\title{
ON THE SUM OF POWERS OF TWO $k$-FIBONACCI NUMBERS WHICH BELONGS TO THE SEQUENCE OF $k$-LUCAS NUMBERS
}

\author{
PAVEl TrojovskÝ
}

\begin{abstract}
Let $k \geq 1$ and denote $\left(F_{k, n}\right)_{n} \geq 0$, the $k$-Fibonacci sequence whose terms satisfy the recurrence relation $F_{k, n}=\bar{k} F_{k, n-1}+F_{k, n-2}$, with initial conditions $F_{k, 0}=0$ and $F_{k, 1}=1$. In the same way, the $k$-Lucas sequence $\left(L_{k, n}\right)_{n \geq 0}$ is defined by satisfying the same recurrence relation with initial values $L_{k, 0}=2$ and $L_{k, 1}=k$. These sequences were introduced by Falcon and Plaza, who showed many of their properties, too. In particular, they proved that $F_{k, n+1}+F_{k, n-1}=$ $L_{k, n}$, for all $k \geq 1$ and $n \geq 0$. In this paper, we shall prove that if $k \geq 1$ and $F_{k, n+1}^{s}+F_{k, n-1}^{s} \in\left(L_{k, m}\right)_{m \geq 1}$ for infinitely many positive integers $n$, then $s=1$.
\end{abstract}

\section{Introduction}

Let $\left(F_{n}\right)_{n \geq 0}$ be the Fibonacci sequence (or sequence of Fibonacci numbers) given by $F_{n+2}=F_{n+1}+F_{n}$, for $n \geq 0$, where $F_{0}=0$ and $F_{1}=1$. The Lucas sequence $\left(L_{n}\right)_{n \geq 0}$ is defined by the same recurrence relation but with seeds $L_{0}=2$ and $L_{1}=1$. The Fibonacci and Lucas numbers are well-known for possessing wonderful and amazing properties (cf. [5] and [9] together with their very extensive annotated bibliography for additional references and history). For instance, the ratio of two consecutive of these numbers converges to the Golden section $\alpha=(1+\sqrt{5}) / 2$. The applications of Golden ratio appear in many research areas, particularly in Physics, Engineering, Architecture, Nature and Art. Material engineer S h e c h t m a n et al. [10] discovered the icosahedral phase in the connection to Golden ratio and aperiodic mosaics, what opened the new field of quasiperiodic crystals. Physicists $\mathrm{N}$ a s c h i e and $\mathrm{M}$ a r ek- $\mathrm{Cr} n$ j a c gave some examples of the Golden ratio in Theoretical Physics and Physics of High

(C) 2016 Mathematical Institute, Slovak Academy of Sciences. 2010 Mathematics Subject Classification: 11D45, 11D61, 11 B39.

Keywords: $k$-Fibonacci number, $k$-Lucas number, Galois theory, Diophantine equation. The research was supported by Specific Research Project of Faculty of Science, University of Hradec Kralove, No. 2101, 2017. 


\section{PAVEL TROJOVSKÝ}

Energy Particles [6], 8]. There are many identities related to Fibonacci and Lucas numbers, e.g., we can cite

$$
F_{n+1}+F_{n-1}=L_{n}, \quad \text { for all } n \geq 0 .
$$

Let $k \geq 1$ and denote $\left(F_{k, n}\right)_{n \geq 0}$, the $k$-Fibonacci sequence whose terms satisfy the recurrence relation

$$
F_{k, n}=k F_{k, n-1}+F_{k, n-2},
$$

with initial conditions $F_{k, 0}=0$ and $F_{k, 1}=1$.

In the same way, the companion $k$-Lucas sequence $L^{(k)}=\left(L_{k, n}\right)_{n \geq 0}$ is defined by satisfying the same recursive relation with initial values $L_{k, 0}=2$ and $L_{k, 1}=k$.

The above sequences are among the several generalizations of Fibonacci and Lucas numbers (case $k=1$ ) and they were extensively studied in the series of papers due to $\mathrm{Falcon}$ and $\mathrm{Plaz}$ a [1]-4]. In particular, they proved that

$$
F_{k, n+1}+F_{k, n-1}=L_{k, n}, \quad \text { for all } n \geq 0 .
$$

In this paper, we shall search for higher power identities related to (1.2) in the spirit of the Marques and Togbé paper [7]. More precisely, we will prove that

Theorem 1.1. Let $k, m$ and $s$ be any positive integers. If $F_{k, n+1}^{s}+F_{k, n-1}^{s} \in$ $\left(L_{k, m}\right)_{m \geq 1}$ is satisfied for infinitely many positive integers $n$, then $s=1$.

\section{Proof of Theorem 1.1}

\subsection{Auxiliary results}

In this section, we shall provide some useful results in order to prove our theorem.

Similarly to the Fibonacci and Lucas sequences, their $k$ versions also satisfy Binet's formulas

$$
F_{k, n}=\frac{\sigma_{1}^{n}-\sigma_{2}^{n}}{\sigma_{1}-\sigma_{2}} \quad \text { and } \quad L_{k, n}=\sigma_{1}^{n}+\sigma_{2}^{n}
$$

where $\sigma_{1}=\left(k+\sqrt{k^{2}+4}\right) / 2$ and $\sigma_{2}=\left(k-\sqrt{k^{2}+4}\right) / 2$. Moreover, note that

$$
\sigma_{1}^{2}=k \sigma_{1}+1 \text { and } \sigma_{1} \sigma_{2}=-1 \text {. }
$$

Falcon and Plaza 2] contains this result

$$
\lim _{n \rightarrow \infty} \frac{F_{k, n+\ell}}{F_{k, n}}=\sigma_{1}^{\ell}
$$

We will need this generalization. 
Lemma 2.1. Let $\alpha, \beta, \gamma, \delta, s, t$ be any positive integers. Then

$$
\lim _{n \rightarrow \infty} \frac{F_{k, \alpha n+\beta}^{s}}{L_{k, \gamma n+\delta}^{t}}=\left\{\begin{array}{cc}
0, & s \alpha-t \gamma<0 ; \\
\frac{\sigma_{1}^{s \beta-t \delta}}{\left(\sigma_{1}-\sigma_{2}\right)^{s}}, & s \alpha-t \gamma=0 ; \\
\infty, & s \alpha-t \gamma>0
\end{array}\right.
$$

and

$$
\lim _{n \rightarrow \infty} \frac{F_{k, \alpha n+\beta}^{s}}{F_{k, \gamma n+\delta}^{t}}=\left\{\begin{array}{cl}
0, & s \alpha-t \gamma<0 \\
\frac{\sigma_{1}^{s \beta-t \delta}}{\left(\sigma_{1}-\sigma_{2}\right)^{s-t}}, & s \alpha-t \gamma=0 \\
\infty, & s \alpha-t \gamma>0
\end{array}\right.
$$

P r o of. Using Binet's formulas for $k$-Fibonacci and $k$-Lucas numbers and subsequently binomial theorem (since $\sigma_{2}<\sigma_{1}$ and $\left|\sigma_{2}\right|<1 / 2$ for $k \geq 2$ ) we have

$$
\frac{F_{k, \alpha n+\beta}^{s}}{L_{k, \gamma n+\delta}^{t}}=\frac{\left(\frac{\sigma_{1}^{\alpha n+\beta}-\sigma_{2}^{\alpha n+\beta}}{\sigma_{1}-\sigma_{2}}\right)^{s}}{\left(\sigma_{1}^{\gamma n+\delta}+\sigma_{2}^{\gamma n+\delta}\right)^{t}}=\left(\sigma_{1}-\sigma_{2}\right)^{-s} \frac{\sigma_{1}^{s(\alpha n+\beta)}+O\left(\sigma_{1}^{(s-1)(\alpha n+\beta)}\right)}{\sigma_{1}^{t(\gamma n+\delta)}+O\left(\sigma_{1}^{(t-1)(\gamma n+\delta)}\right)}
$$

and

$$
\frac{F_{k, \alpha n+\beta}^{s}}{F_{k, \gamma n+\delta}^{t}}=\frac{\left(\frac{\sigma_{1}^{\alpha n+\beta}-\sigma_{2}^{\alpha n+\beta}}{\sigma_{1}-\sigma_{2}}\right)^{s}}{\left(\frac{\sigma_{1}^{\gamma n+\delta}-\sigma_{2}^{\gamma n+\delta}}{\sigma_{1}-\sigma_{2}}\right)^{t}}=\left(\sigma_{1}-\sigma_{2}\right)^{t-s} \frac{\sigma_{1}^{s(\alpha n+\beta)}+O\left(\sigma_{1}^{(s-1)(\alpha n+\beta)}\right)}{\sigma_{1}^{t(\gamma n+\delta)}+O\left(\sigma_{1}^{(t-1)(\gamma n+\delta)}\right)} .
$$

Let us consider $n \rightarrow \infty$. Thus, one has

$$
O\left(\sigma_{1}^{(t-1)(\gamma n+\delta)}\right) / \sigma_{1}^{t(\gamma n+\delta)} \rightarrow 0 \quad \text { and } \quad \sigma_{1}^{s(\alpha n+\beta)} / \sigma_{1}^{t(\gamma n+\delta)} \rightarrow 0
$$

if and only if $s \alpha-t \gamma=0$. Therefore the assertions easily follow.

Lemma 2.2. For all $k \geq 1$ and $n \geq 1$, it holds that

$$
\sigma_{1}^{n-2} \leq F_{k, n} \leq \sigma_{1}^{n-1} \quad \text { and } \quad \sigma_{1}^{n-1}<L_{k, n}<\sigma_{1}^{n+1} \text {. }
$$

P r o of. In order to avoid unnecessary repetitions, we shall prove only that $F_{k, n} \leq \sigma_{1}^{n-1}$ (the other inequalities are proved in the same way). The proof is by induction on $n$. For the basic step note that

$$
F_{k, 1}=1 \leq \sigma_{1}^{1-1} \quad \text { and } \quad F_{k, 2}=k \leq \sigma_{1}^{2-1} .
$$

Now suppose, by induction hypothesis, that $F_{k, j} \leq \sigma_{1}^{j-1}$ for all $1 \leq j \leq n$. Then

$$
F_{k, n+1}=k F_{k, n}+F_{k, n-1} \leq k \sigma_{1}^{n-1}+\sigma_{1}^{n-2}=\sigma_{1}^{n-2}\left(k \sigma_{1}+1\right)=\sigma_{1}^{n-2} \cdot \sigma_{1}^{2}=\sigma_{1}^{n}
$$

and the result follows by induction.

Lemma 2.3. Let $k, s$ be any positive integers. Then

$$
L_{k, s} \leq\left(k^{2}+2\right)^{s / 2} .
$$




\section{PAVEL TROJOVSKÝ}

Proof. We use mathematical induction on $s$. For $s=1$ and $s=2$ we have $L_{k, 1} \leq\left(k^{2}+2\right)^{1 / 2}$ and $L_{k, 2} \leq\left(k^{2}+2\right)^{1}$, what easily follows from the $L_{k, 1}=k$ and $L_{k, 2}=k^{2}+2$. For $s \geq 3$ we have

$$
\begin{aligned}
L_{k, s} & =k L_{k, s-1}+L_{k, s-2} \\
& \leq k\left(k^{2}+2\right)^{(s-1) / 2}+\left(k^{2}+2\right)^{(s-2) / 2} \\
& \leq\left(k^{2}+2\right)^{s / 2}\left(k\left(k^{2}+2\right)^{-1 / 2}+\left(k^{2}+2\right)^{-1}\right) \\
& \leq\left(k^{2}+2\right)^{s / 2} \frac{k \sqrt{k^{2}+2}+1}{k^{2}+2} \leq\left(k^{2}+2\right)^{s / 2}
\end{aligned}
$$

as $\frac{k \sqrt{k^{2}+2}+1}{k^{2}+2} \leq 1$ immediately follows from $k \sqrt{k^{2}+2}<k^{2}+1$, which clearly holds for every positive integer $k$.

Now, we are ready to deal with the proof of Theorem 1.1

\subsection{The proof of Theorem 1.1}

Suppose that $F_{k, n+1}^{s}+F_{k, n-1}^{s}=L_{k, t(n)}$ for infinitely many positive integers $n$ (say $n$ belongs to an infinite set $\mathcal{S}$ ), where $t(n)$ is an integer function.

By using the estimates in Lemma 2.2, we shall have

$$
\begin{aligned}
\sigma^{t(n)-1}<L_{k, t(n)} & =F_{k, n+1}^{s}+F_{k, n-1}^{s}<\sigma^{s n}+\sigma^{s(n-2)} \leq \sigma^{s(n-2)}\left(1+\sigma^{2 s}\right) \leq \sigma^{s n+1} \\
\text { and } & \begin{aligned}
\sigma^{t(n)+1}>L_{k, t(n)} & =F_{k, n+1}^{s}+F_{k, n-1}^{s}>\sigma^{s(n-1)}+\sigma^{s(n-3)} \\
& \geq \sigma^{s(n-3)}\left(1+\sigma^{2 s}\right) \geq \sigma^{s(n-3)} \sigma^{2 s} \geq \sigma^{s(n-1)} .
\end{aligned}
\end{aligned}
$$

Thus, $s n-s-1<t(n)<s n+2$ for all $n \in \mathcal{S}$. Therefore, $t(n)=n s+t$, for all $n \in \mathcal{S}^{\prime} \subseteq \mathcal{S}$, where $\mathcal{S}^{\prime}$ is an infinite set and $t$ is a constant, which depends only on $s$. Then, we consider the equation

$$
F_{k, n+1}^{s}+F_{k, n-1}^{s}=L_{k, n s+t}
$$

and divide it by $L_{k, n s+t}$. Therefore,

$$
\frac{F_{k, n+1}^{s}}{L_{k, n s+t}}+\frac{F_{k, n-1}^{s}}{L_{k, n s+t}}=1 .
$$

Now we consider $n \rightarrow \infty$ (in $\mathcal{S}^{\prime}$ ) in the previous equation. Thus, using Lemma 2.1 we have

$$
\begin{aligned}
\lim _{n \rightarrow \infty, n \in \mathcal{S}^{\prime}}\left(\frac{F_{k, n+1}^{s}}{L_{k, n s+t}}+\frac{F_{k, n-1}^{s}}{L_{k, n s+t}}\right) & =\left(\sigma_{1}-\sigma_{2}\right)^{-s} \frac{1}{\sigma_{1}^{t+s}}+\left(\sigma_{1}-\sigma_{2}\right)^{-s} \frac{1}{\sigma_{1}^{t-s}} \\
& =\left(\sigma_{1}-\sigma_{2}\right)^{-s} \frac{1}{\sigma_{1}^{t+s}}\left(1+\sigma_{1}^{2 s}\right)
\end{aligned}
$$


Hence, we obtain the Diophantine equation

$$
1+\sigma_{1}^{2 s}=\left(\sqrt{k^{2}+4}\right)^{s} \sigma_{1}^{s+t} .
$$

Marques and Togbé [7] found lower bounds for linear forms in logarithms to solve similar equation for Fibonacci numbers. But this approach is not usable, as the equation (2.3) is not purely exponential (because of the $k$ in the basis). Instead of linear forms, we shall use a little taste of algebraic and Galois theory.

Now, note that $\sigma_{1}, \sigma_{2} \in \mathbb{K}:=\mathbb{Q}\left(\sqrt{k^{2}+4}\right)$, then by conjugating the relation (2.3) by the automorphism $\psi: \sigma_{1} \mapsto \sigma_{2}$ of $\operatorname{Gal}(\mathbb{K} / \mathbb{Q})$, we obtain

$$
1+\sigma_{2}^{2 s}=\left(-\sqrt{k^{2}+4}\right)^{s} \sigma_{2}^{s+t} .
$$

By multiplying (2.3) by (2.4) and by using that $\sigma_{1} \sigma_{2}=-1$, we obtain

$$
1+(-1)^{2 s}+\sigma_{1}^{2 s}+\sigma_{2}^{2 s}=(-1)^{2 s+t}\left(k^{2}+4\right)^{s},
$$

which can be rewritten, using Binet's formula for $k$-Lucas numbers and using a clear condition that $t$ has to be even, by the following way

$$
L_{k, 2 s}=\left(k^{2}+4\right)^{s}-2 .
$$

For $s=1$ the last equality holds for any $k$. For $s \geq 2$ with respect to Lemma 2.3 and the clear fact that

$$
\left(k^{2}+2\right)^{s}<\left(k^{2}+4\right)^{s}-2
$$

holds for every positive integers $k, s$, we see that the solution does not exist. The proof is then complete.

\section{REFERENCES}

[1] FALCON, S.-PLAZA, A.: On the Fibonacci $k$-numbers, Chaos Solitons Fractals 32 (2007), 1615-1624.

[2] FALCON, S.-PLAZA, A.: The $k$-Fibonacci sequence and the Pascal 2-triangle, Chaos Solitons Fractals 33 (2007), 38-49.

[3] FALCON, S.-PLAZA, A.: On k-Fibonacci numbers of arithmetic indexes, Appl. Math. Comput. 208 (2009), 180-185.

[4] FALCON, S.-PLAZA, A.: Binomial transforms of the k-Fibonacci sequence, Int. J. Nonlinear Sci. Numer. Simul. 10 (2009), 1527-1538.

[5] KALMAN, D.-MENA, R.: The Fibonacci numbers exposed, Math. Mag. 76 (2003), $167-181$.

[6] MAREK-CRNJAC, L.: On the mass spectrum of the elementary particles of the standard model using El Naschie's golden field theory, Chaos Solitons Fractals 15 (2003), $611-618$.

[7] MARQUES, D.-TOGBÉ, A.: On the sum of powers of two consecutive Fibonacci numbers, Proc. Japan Acad. Ser. A 86 (2010), 174-176.

[8] EL NASCHIE, M. S.: The golden mean in quantum geometry, Knot theory and related topics, Chaos Solitons Fractals 10 (1999), 1303-1307. 


\section{PAVEL TROJOVSKÝ}

[9] POSAMENTIER, A.S.-LEHMAN, I.: The (Fabulous) Fibonacci Numbers, Prometheus Books, Amherst, NY, 2007.

[10] SHECHTMAN, D.-BLECH, I.-GRATIAS, D.-CAHN, J. W.: Metallic phase with long-range orientational order and no translational symmetry, Phys. Rev. Lett. 53 (1984), 1951-1953.

Received September 30, 2016

Departament of Mathematics Faculty of Natural Sciences University of Hradec Králové Hradecká 1285

CZ-500-03 Hradec Králové CZECH REPUBLIC

E-mail: Pavel.Trojovsky@uhk.cz 